\title{
Model-Free Design Optimisation of a Hopping Robot and Its Comparison with a Human Designer
}

\author{
Kaur Aare Saar ${ }^{1}$, Fabio Giardina ${ }^{1}$, and Fumiya Iida ${ }^{1}$
}

\begin{abstract}
When developing a robot, design iterations in the physical world are necessary, even though they are often costly and not systematic. Here we present an automated iterative design process without using modelling or simulation, which we refer to as "model-free design optimisation" based on Bayesian optimisation. This paper particularly focuses on the co-optimisation of morphology and controller, by using a mechanism to balance parameter specific costs (i.e. morphology samplings are more expensive than control ones) for effective and efficient design optimisation processes. A hopping robot was employed for a feasibility analysis of the proposed optimisation method, in which minimalistic two-dimensional and fourdimensional design optimisation experiments were performed in real life. The results show that the proposed approach is capable of improving both of the robot design problems within a defined time limit. The method is also compared to optimisation performances of a human designer under the same conditions. While the human optimisation performs better in the 2-dimensional search space, the automated method found the best solution quicker in the 4-dimensional case.
\end{abstract}

\section{INTRODUCTION}

Development of robotic systems generally requires many design iterations in the real world. Mathematical modelling and computer simulations are helpful tools, but development and evaluation of robots in real life are inevitable for many applications that cannot be accurately simulated, despite the high economical and temporal costs. This paper investigates how real-world design iterations of robots can be systematically performed by using automated optimisation techniques, which we refer to as "model-free design optimisation".

The use of optimisation algorithms to design robots has been studied intensively in the past, particularly in the context of robot locomotion [1], [2], [3], which is the focus of this paper. The main body of literature reports on optimisation methods for locomotion controllers by using, for example, reinforcement learning [4], nonlinear optimisation [5], or evolutionary algorithms [6]. Focus is commonly placed on the optimisation of control architectures, which is interesting and important on its own right, but there has been an increasing interest of co-optimisation of morphology (mechanical structure) and control simultaneously. Cooptimisation of morphology and control is of fundamental importance, as the robot dynamics only result as a function of both design aspects. Significant work of co-optimisation is due to Sims [7] and Lipson [8], which use evolutionary algorithms for optimisation, and the real-life optimisation as presented in [9], [10], [11].

\footnotetext{
${ }^{1}$ Bio-Inspired Robotics Lab, Department of Engineering, University of Cambridge Trumpington Street, Cambridge CB2 1PZ, United Kingdom [ffg20, fi224] @cam.ac.uk
}

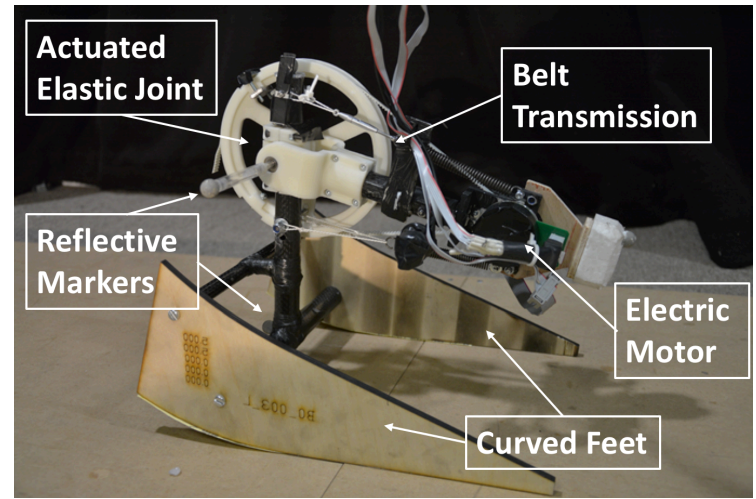

Fig. 1: Hopping robot CHIARO.

From this perspective, this paper proposes the use of datadriven optimisation algorithm, Bayesian optimisation, for the problems of morphology-controller co-optimisation. The algorithm is known as one of the most efficient methods to optimise black-box functions, especially for the problems where sampling is expensive. Unlike the conventional model-based robot design optimisation such as [12], this approach allows optimisation without explicit mathematical modelling, and the process can take place in real life. The approach was previously used to optimise locomotion robot controllers [13], [9], but it was either not applied for co-optimisation of morphology and control or only for crawling-like locomotion. Therefore we apply this method to the design optimisation of both morphology and controller of highly dynamical locomotion robots, and evaluate the degrees to which the algorithm can handle the expensive samplings. As the case study, we employ a hopping robot which we have been developing in the past years [14] and is shown in Fig. 1 Prior to optimisation, we restrict the parameter dimensionality of morphological and controller designs, and let the algorithm explore the design space for better locomotion performance.

One of the most important considerations in this framework is to properly maintain the balance between exploration of morphological parameters and that of controllers, because the former sampling is significantly more expensive than the latter. For this reason, this paper proposes a mechanism, that we call "Dynamic Scheduling", which considers parameter specific costs within Bayesian optimisation dynamically for each iteration. As it becomes clearer later in this paper, this mechanism turns out to be crucial for the design optimisation of dynamic robot, which are shown through the case study.

The rest of paper is structured as follows. First, we 
introduce the model-free robot design Optimisation. Second, we explain how this framework can be implemented for a comprehensive test, which is followed by experimental results and analysis. Finally, we discuss the implications and future work.

\section{MODEL-FREE DESIGN OPTIMISATION}

\section{A. Bayesian optimisation Algorithm}

In our task of optimising a complex dynamical system subject to effects that are challenging to model (e.g. environment interactions), optimisation algorithms that don't rely on an accurate model are the preferred choice. Bayesian optimisation ${ }^{1}$ is such a method and can sample efficiently from black-box functions where gradient information is not easily obtained. It is a method employed to minimise the number of iterations required to find the maximum of an objective function $f(\boldsymbol{\theta})$, where $\boldsymbol{\theta}$ is the parameter vector. Efficient sampling is crucial if sampling is expensive - which is commonly the case in real-world design iterations. We are interested in finding the parameter vector which maximises the objective function, i.e. $\boldsymbol{\theta}^{*}=\max _{\boldsymbol{\theta}} f(\boldsymbol{\theta})$.

1) Gaussian process model: Assuming the objective function is already evaluated at $n$ points with argument values $\boldsymbol{\theta}_{1: n} \equiv\left[\boldsymbol{\theta}_{1}, \ldots, \boldsymbol{\theta}_{n}\right]^{T}$ and function values $\boldsymbol{f}_{1: n} \equiv$ $\left[f\left(\boldsymbol{\theta}_{1}\right), \ldots, f\left(\boldsymbol{\theta}_{n}\right)\right]^{T}$, these points are used as training to determine the parameters for their covariance function. We employ a typical Matérn kernel (with smoothness parameter $\varsigma=3 / 2$ ) as defined in [15]. For points $\boldsymbol{\theta}_{i}$ and $\boldsymbol{\theta}_{j}$ the covariance can be calculated as:

$$
k\left(\boldsymbol{\theta}_{i}, \boldsymbol{\theta}_{j}\right)=\sigma_{f}^{2}(1+\sqrt{3} r) \exp (-\sqrt{3} r)
$$

where

$$
r=\sqrt{\sum_{d=1}^{n} \frac{\left(\theta_{i d}-\theta_{j d}\right)^{2}}{l_{d}^{2}}}
$$

The hyperparameters of Matérn kernel with automatic relevance detection are $\sigma_{f}$ i.e. signal variance and $l_{d}$ i.e. the lengthscale hyperparameter for $d$-th model parameter.

For each pair of training points, the covariance function is evaluated and arranged to a matrix as follows:

$$
\mathbf{K}=\left[\begin{array}{cccc}
k\left(\boldsymbol{\theta}_{1}, \boldsymbol{\theta}_{1}\right) & k\left(\boldsymbol{\theta}_{1}, \boldsymbol{\theta}_{2}\right) & \ldots & k\left(\boldsymbol{\theta}_{1}, \boldsymbol{\theta}_{n}\right) \\
k\left(\boldsymbol{\theta}_{2}, \boldsymbol{\theta}_{1}\right) & k\left(\boldsymbol{\theta}_{2}, \boldsymbol{\theta}_{2}\right) & \ldots & k\left(\boldsymbol{\theta}_{2}, \boldsymbol{\theta}_{n}\right) \\
\vdots & \vdots & \ddots & \vdots \\
k\left(\boldsymbol{\theta}_{n}, \boldsymbol{\theta}_{1}\right) & k\left(\boldsymbol{\theta}_{n}, \boldsymbol{\theta}_{2}\right) & \ldots & k\left(\boldsymbol{\theta}_{n}, \boldsymbol{\theta}_{n}\right)
\end{array}\right]
$$

The hyperparameters are found by maximising their marginal likelihood which, according to Bayes theorem, is the same as maximising the marginal likelihood of the training data given the model parameters. This is done using local iterative gradient-based optimisation methods as described in [15]. Once the hyperparameters that explain the

${ }^{1}$ More details of the algorithm can be found in our previous work published in the repository: https://WwW mathworks.com/matlabcentral/fileexchange/ 59060-bayesian-optimisation-of-slip-model-parameter s_tid=srchtitle training data the best have been found, they are used to predict the expected values $\mu\left(\boldsymbol{\theta}_{k}\right)$ and the confidence $\sigma\left(\boldsymbol{\theta}_{k}\right)$ of any point $\boldsymbol{\theta}_{k}$ in the parameter space as follows.

$$
\begin{aligned}
\mu\left(\boldsymbol{\theta}_{k}\right) & =\mathbf{k}_{k}^{T} \mathbf{K}^{-1} \boldsymbol{f}_{1: n} \\
\sigma^{2}\left(\boldsymbol{\theta}_{k}\right) & =-\mathbf{k}_{k}^{T} \mathbf{K}^{-1} \mathbf{k}_{k}
\end{aligned}
$$

where $\mathbf{k}_{k}=\left[k\left(\boldsymbol{\theta}_{1}, \boldsymbol{\theta}_{k}\right), \ldots, k\left(\boldsymbol{\theta}_{n}, \boldsymbol{\theta}_{k}\right)\right]^{T}$. The process of training the hyperparameters and drawing predictions from the Gaussian process is repeated every time as a new point is sampled.

2) Acquisition function: The acquisition function that we use in this paper is the expected improvement (EI) which is calculated by integrating the improvement over the probability density of the prediction as in [16].

$$
\begin{aligned}
\operatorname{EI}(\boldsymbol{\theta}) & =\int_{0}^{\infty} I \cdot \mathcal{N}\left(\mu(\boldsymbol{\theta})-\max \left(\boldsymbol{f}_{1: n}\right)-\xi-I, \sigma(\boldsymbol{\theta})\right) d I \\
& =\left(\mu(\boldsymbol{\theta})-\max \left(\boldsymbol{f}_{1: n}\right)-\xi\right) \Phi(Z)+\sigma(\boldsymbol{\theta}) \phi(Z)
\end{aligned}
$$

where

$$
Z=\frac{\mu(\boldsymbol{\theta})-\max \left(\boldsymbol{f}_{1: n}\right)-\xi}{\sigma(\boldsymbol{\theta})}
$$

and $\Phi(Z)$ and $\phi(Z)$ denote the cumulative density function and probability density function of the standard normal distribution respectively [16]. It is important to note that probability of improvement is very sensitive to the choice of the trade-off parameter $\xi$. If $\xi$ is too small, the function will only be sampled closed to its existing maximum value, whereas if $\xi$ is too large, it will only be sampled in the regions where the least points have been sampled. Expected improvement is less sensitive and [17] suggests that the trade-off parameter could be set to $\xi=0.01$ throughout the optimisation for the best results.

Finally, to find the next sampling point, the acquisition function is maximised.

$$
\boldsymbol{\theta}_{n+1}=\underset{\boldsymbol{\theta}}{\operatorname{argmax}} \mathrm{EI}(\boldsymbol{\theta})
$$

Though this equation introduces another optimisation problem, the evaluation of these values is sufficiently cheap. In this project $10^{5}$ points are placed on the grid according to the significance of the hyperparameters, i.e. the spacing of the sampling grid is proportional to the lengthscale hyperparameter in all directions.

\section{B. Robot Platform}

To test the model-free design optimisation we employ the hopping robot called CHIARO, which we have been investigating in many of our previous projects [14], [20], [18], [19]. The robot consists of two hinged bodies which are connected with springs as shown in Fig. 11. The robot exhibits hopping locomotion when torque is applied between the bodies. The resonant frequency of the system is exploited to create a forward hopping motion with minimal energy input. CHIARO was first modelled and constructed in [20], which had a circular foot shape and was controlled using 

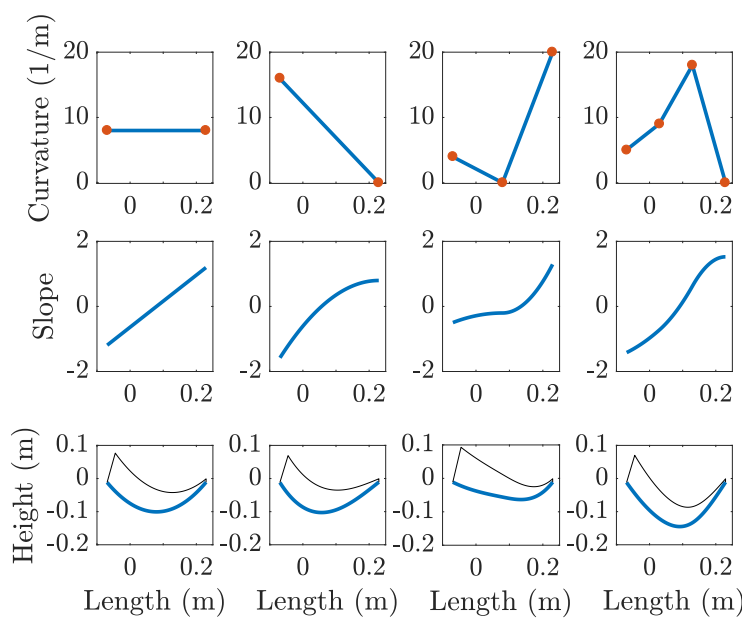

(a)

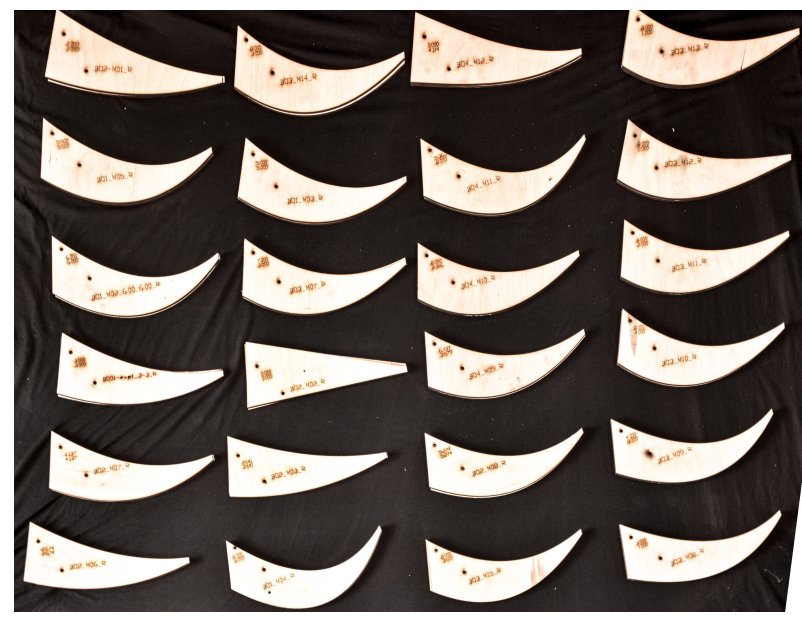

(b)

Fig. 2: (a) Morphology parameters are defined as curvatures at discrete points on the foot, and the foot shape is derived through integration of the curvature resulting in $2 \mathrm{D}$ foot shapes in a Height-Length domain. (b) Foot shape samples which were designed by the model-free design optimisation for improved locomotion speed in the robot.

a sinusoidal input at its resonance frequency. It was found that locomotion speeds can be maximised when an openloop pulse torque input is given to the motor of the robot [19], which we employ in the experiments of this paper. Even though the earlier work showed that foot shapes in general has an effect of the stability and speed of hopping locomotion [14], [18], no systematic investigations were made to analyse the performance with more complex foot shapes.

To parametrise CHIARO's foot shape, we define its curvature at discrete points along the foot. The number of defined curvatures is $m$, each placed uniformly over the horizontal axis of the foot with the length $L=30 \mathrm{~cm}$. The curvature along the rest of the foot shape is interpolated linearly between the discrete points. Integrating the curvature twice results in a foot shape as shown in Fig. 2 for $m=1,2,3,4$.
Additional sides are added to provide mounting points to CHIARO's body. We only allow for non-negative curvatures to obtain a convex shape. The integration constants are chosen such that the heel and the toe of the feet are on the same level to account for the attachment to the robot's main body. Some foot shapes that were built during the experiments are shown in Fig. 2(b).

\section{EXPERIMENTAL METHOD}

\section{A. Experiment procedure}

Experiments of model-free design optimisation start by defining an objective function and robot design parameters. Once the parameter landscape is set, the remaining procedure is automated as shown in Fig. 3 Namely the morphology and control parameters were automatically determined based on the algorithm, and then the robot platform is configured for testing. When morphology is chosen to be changed, new feet are laser-cut and assembled into the robot body, which usually requires approximately 20 minutes in total. The robot is connected to a computer over a USB interface and the target torque input is controlled using a MATLAB script. The robot is then placed in a locomotion track as shown in Fig. 3. The track has railings on each side to ensure consistency of the robot motion. The track is covered with a rubber mat for improved traction between CHIARO and the floor. Both, the body and the leg of CHIARO, are equipped with 4 trackable markers which are recorded by 12 infrared cameras of a motion capturing system to deduce locomotion speed. With each set of chosen parameters, 3 measurements are made and the average speed of them is taken as the true value. Each run is limited to $t=10 \mathrm{~s}$ and the average speed is calculated by comparing the initial and final position of CHIARO. The outcome of this experiment is then given to the optimisation algorithm to obtain the next sampling point. For each experiment below, we set the time limit to 10 hours with the possibility of earlier termination when saturated. From the start of each experiment, we counted the duration of every relevant process including the time for laser cutting the feet, as well as locomotion experiments and computation time of the optimisation algorithm.

\section{B. Choice of design parameters}

The choice of design parameters substantially influences the experimental results. We are interested in co-optimising morphology and control, which requires at least a 2dimensional parameter space. We conducted two sets of experiments to test different dimensionality. In the first set of experiments, the algorithm optimises in a 2-dimensional parameter space, i.e. the curvature of the foot, and an actuation frequency of motor oscillation, $\theta_{M}=\kappa, \theta_{C}=$ $f$. The choice of parameters was affected by both: the theoretical expected influence on the model and feasibility of implementation. In the second set of experiments, we extend the parameter space dimension to 4, i.e. two parameters for the foot curvature (defining curvature at the toe and the heel), and two parameters for motor control. The second control parameter, in addition to the actuation frequency, is the duty 


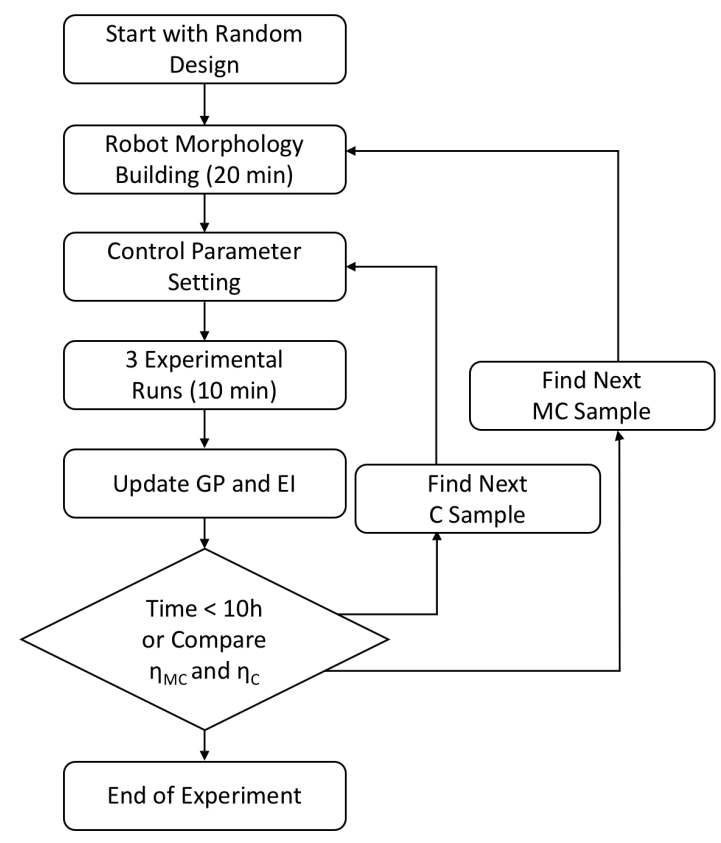

(a) Flowchart

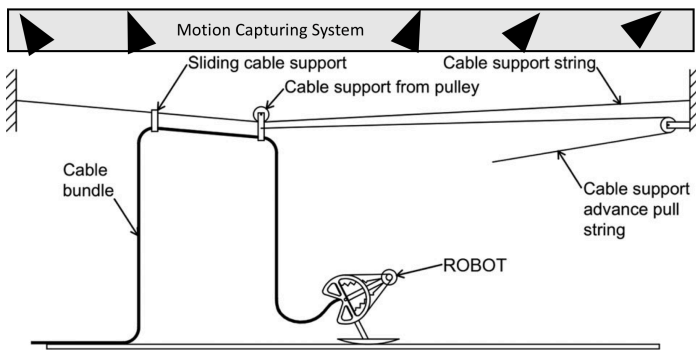

(b) Experimental Setup

Fig. 3: (a) Flowchart summarising the model-free design optimisation in real-world, and (b) an illustration of experimental setup for the hopping robot CHIARO.

cycle of the pulse wave. The parameter vectors are then $\theta_{M}=\left[\begin{array}{c}\kappa_{1} \\ \kappa_{2}\end{array}\right]$ and $\theta_{C}=\left[\begin{array}{l}f \\ \lambda\end{array}\right]$. All other parameters were fixed to the values based on our previous research [19], e.g. the duty cycle of the motor oscillation to be set at $\lambda=0.25$ (in the first set of experiments) and the target torque amplitude at $T=80 \mathrm{mNm}$.

\section{Dynamic scheduling}

Another parameter selection has to be made between morphology and control, because the cost of samplings (time requires to test) is significantly different. In our experimental setup, the time to evaluate different morphology is approximately three times longer $(30 \mathrm{~min})$ than the control only (10 min). This information has to be provided to the Bayesian optimisation procedure in order to maximise the improvement over the limited time, as a similar procedure was proposed in [21]. For this reason, we introduce two variables $\eta_{M C}$ and $\eta_{C}$, which indicate expected improvement of morphology- control optimisation and that of control optimisation only, respectively:

$$
\begin{aligned}
& \eta_{M C}=\max _{\theta_{M}, \theta_{C}}\left(\operatorname{EI}\left(\left[\begin{array}{c}
\theta_{M} \\
\theta_{C}
\end{array}\right]\right)\right) \\
& \eta_{C}=\max _{\theta_{C}}\left(\operatorname{EI}\left(\left[\begin{array}{c}
\theta_{M}^{\text {current) }} \\
\theta_{C}
\end{array}\right]\right)\right)
\end{aligned}
$$

With these two variables, the algorithm determines to perform control parameter optimisation when $\zeta \eta_{C}>\eta_{M C}$, where $\zeta$ is a correction factor to represent the cost of sampling (therefore we set $\zeta=\frac{30 \mathrm{~min}}{10 \mathrm{~min}}=3$ for all the experiments below). Conversely, optimisation of both morphology and control parameters is performed when the above inequality is false.

\section{Experiment with a human designer procedure}

For the purpose of benchmarking, the proposed optimisation method was compared to another set of experiments in which a human designer was instructed to optimise locomotion speed of CHIARO in the exactly same conditions. The human designer has an engineering background and has previously used the experimental platform, but not for optimisation of morphology and control. Unlike Bayesian optimisation, the human designer has access to his experiences and can analyse the motion capturing data to decide on the next sampling point. Although the human designer would have advantages in the sampling strategies because of the prior knowledge and more information about the experiments, we performed the comparative study as fairly as possible. For this reason, the experiments were performed with the human designers first, which is then followed by the Bayesian optimisation.

\section{EXPERIMENTAL RESULTS}

\section{A. Optimisation speed}

The locomotion speed of CHIARO in each trial was plotted in Fig. 4 along with the cumulative maximum values. The speed improvement is shown both for the 2-dimensional experiments and the 4-dimensional ones. Note that the prior to the 4-dimensional optimisation was the information gained from the 2-dimensional experiment, because the human designer had a similar advantage. The figure shows that in the 2-dimensional experiments, both human and machine converged to the final speed of $v=0.26 \mathrm{~m} \mathrm{~s}^{-1}$ which is believed to be the maximum value within the given search range. However, the human designer outperforms the Bayesian optimisation procedure in terms of time to find the maximal locomotion speed. While the human designer reaches the maximum value within 3 hours, Bayesian optimisation reached the maximum speed after 6 hours. This can be understood from the fact that Bayesian optimisation needed to explore the search space to a greater extent than the human designer, as the latter had access to motion data and prior experience.

In the 4-dimensional experiments, in contrast, Bayesian optimisation outperforms the human designer. While the 


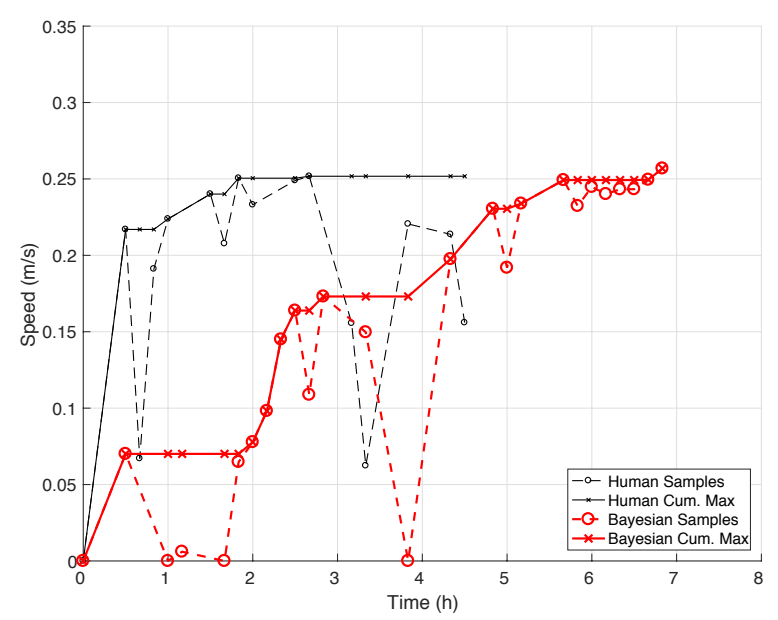

(a) 2D Experiments

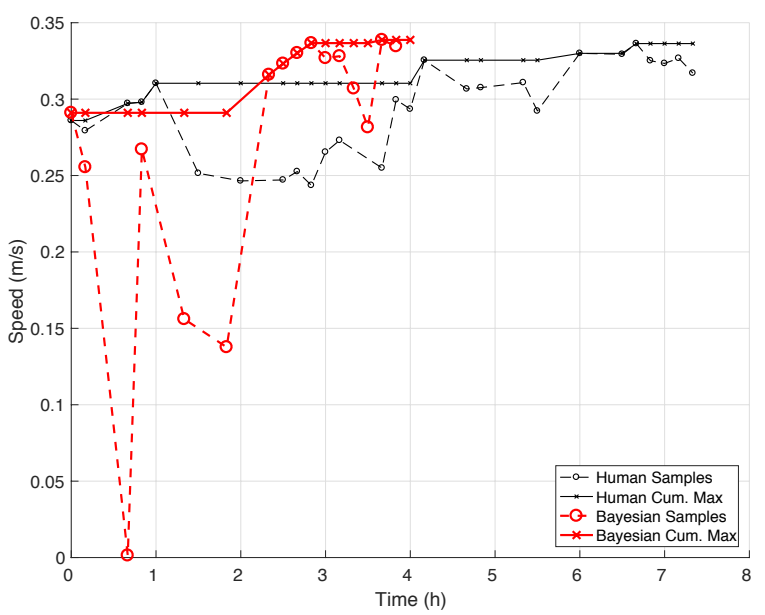

(b) 4D Experiments

Fig. 4: Comparison of optimisation process for the human designer and the model-free design optimisation. Results are shown for the 2-dimensional and 4-dimensional parameter space.

human designer needed almost 7 hours to find the highest locomotion speed at $0.336 \mathrm{~m} \mathrm{~s}^{-1}$, the Bayesian optimisation reached to similar highest speed $0.339 \mathrm{~m} \mathrm{~s}^{-1}$ in less than 4 hours (Table III). We ascribe this result to the fact that Bayesian optimisation started with the prior from the 2dimensional case and had, hence, a fair representation of the larger parameter space.

\section{B. Analysis of sampling strategies}

Fig. 5 shows the sampling points in the 2-dimensional parameter space together with the final Gaussian process model in both human and Bayesian optimisation. The results show that while the human designer quickly started narrowing down the best parameter regions, the Bayesian optimisation explored one morphology (curvature $2 \mathrm{~m}^{-1}$ ) in particular. This contrast in the exploration strategies could be

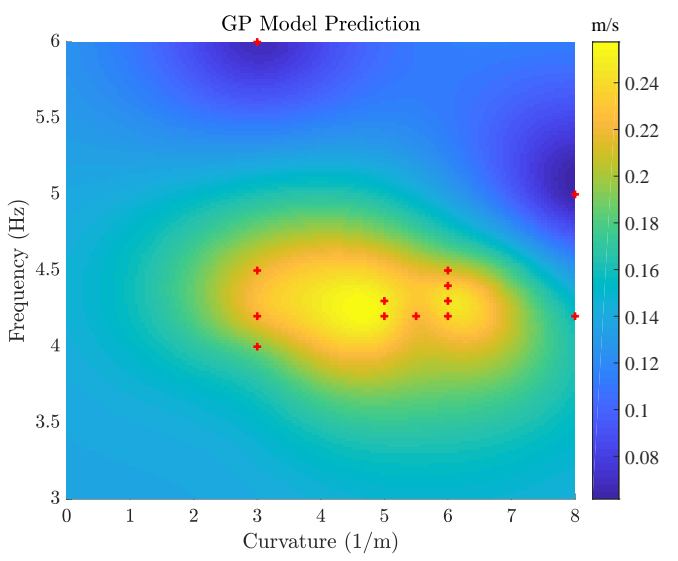

(a) Human Designer

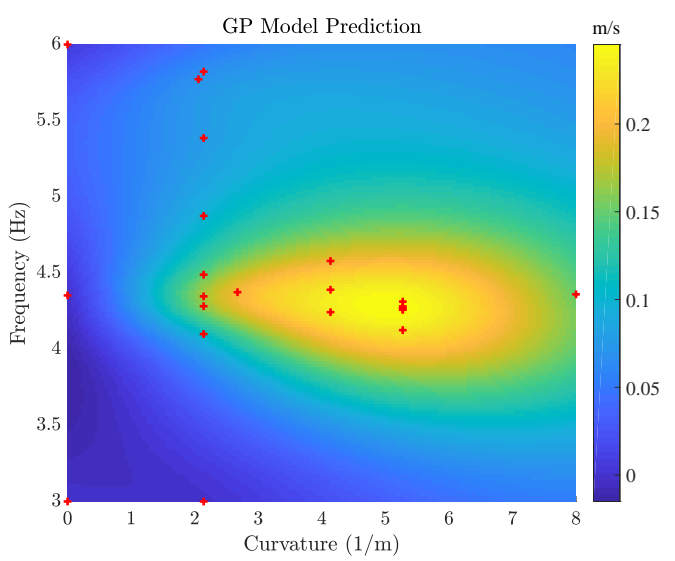

(b) Bayesian Optimisation

Fig. 5: Gaussian Process model prediction of the locomotions speed and expected improvement for (a) a human designer, and (b) the model-free design optimisation. The red crosses indicate the parameter sampling points (corresponding to a frequency/curvature pair) in the real-world robot for the 2dimensional parameter space experiment.

understood from the fact that the human designer had a better intuition about the influence of frequency to the behaviour of the hopping robot. In contrast, the Bayesian optimisation explores the parameter space based on the correction factor $\zeta$, which accounts for parameter-specific sampling costs. The 8 sampling points with the same curvature amounted for 80 minutes of experiments, while 8 points with changing curvature would have caused 240 minutes of experiments. This strategy can be understood as the effect of dynamic scheduling that we employed in the algorithm.

In the 4-dimensional parameter space, we found that the human designer and the Bayesian optimisation were exploring and exploiting the empirical knowledge in a similar manner. Fig. 6 shows the parameters to be explored during the experiments. Both the human and Bayesian optimisation explored similar regions of morphology, whereas sampling of the control parameters differs with respect to the frequency similar to the 2-dimensional experiments. This suggests that 


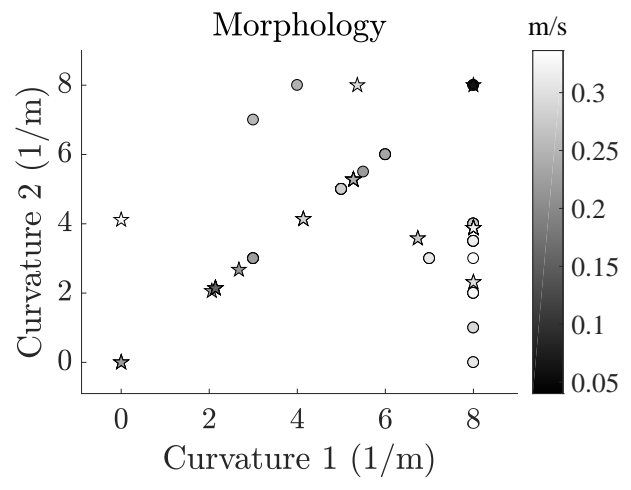

(a) Morphology Parameters

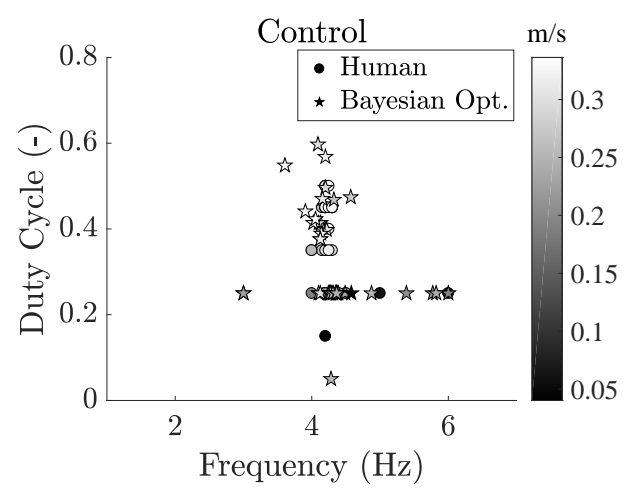

(b) Control Parameters

Fig. 6: Sampling behaviours of the human designer and the Bayesian optimisation in the 4-dimensional experiment, plotted on the parameter spaces of (a) Morphology and (b) Control.

an accurate model of the real system was formed in the Bayesian optimisation, in contrast to the human designer who may have struggled to map the intuition of the system behaviour onto the vastly larger 4-dimensional parameter space.

\section{Parameters and behaviours for best performances}

Finally we also compare the quality of optimisation results by analysing the physical behaviours of the robot. Because the design parameters obtained by the Bayesian optimisation and human design process are similar in both 2-dimensional and 4-dimensional experiments, the locomotion behaviours of the robot are also very similar as shown in Table $\square$ and II

The most important difference between the trajectories of the best model obtained in 2-dimensional and 4-dimensional experiments is the posture. Having two parameters for the curvature of the feet allows to modify the posture at the rest position. Fig. 7 indicates that the rest position in the 4-dimensional experiments tilts forward, which provides a flatter take-off angle at touchdown and hence a faster motion. During the forward motion of the robot, the posture keeps varying and the ground contact points are chaotic throughout
TABLE I: Fastest robot in 2-D optimisation

\begin{tabular}{l|cc}
\hline Parameters & Bayesian & Human \\
\hline \hline$\kappa\left(\mathrm{m}^{-1}\right)$ & 5.3 & 5.0 \\
\hline$f(\mathrm{~Hz})$ & 4.3 & 4.2 \\
\hline$v\left(\mathrm{~m} \mathrm{~s}^{-1}\right)$ & $0.250 \pm 0.003$ & $0.252 \pm 0.003$ \\
\hline Optimisation time (h) & 6.8 & 2.7 \\
\hline
\end{tabular}

TABLE II: Fastest robot in 4-D optimisation

\begin{tabular}{l|cc}
\hline Parameters & Bayesian & Human \\
\hline \hline$\kappa_{1}\left(\mathrm{~m}^{-1}\right)$ & 8.0 & 8.0 \\
\hline$\kappa_{2}\left(\mathrm{~m}^{-1}\right)$ & 3.9 & 4.2 \\
\hline$f(\mathrm{~Hz})$ & 4.14 & 4.25 \\
\hline$\lambda$ & 0.39 & 0.45 \\
\hline$v\left(\mathrm{~m} \mathrm{~s}^{-1}\right)$ & $0.339 \pm 0.006$ & $0.336 \pm 0.001$ \\
\hline Optimisation time $(\mathrm{h})$ & 3.7 & 6.8 \\
\hline
\end{tabular}

the experiments. Even though the locomotion results of both human designer and Bayesian optimisation are very similar, the qualitative behaviours are considerably different, as the Bayesian optimisation found a more non-periodic solution of locomotion, which are usually very difficult for human to optimise.

\section{CONCLUSIONS}

This paper introduced a design optimisation framework of physical robots based on the state-of-the-art Bayesian optimisation algorithm. Design optimisation of physical robots can be characterised by the fact that simulation is often very challenging, thus "model-free approach" is useful. In addition, robot design iterations also require optimisation of both mechanical and control parameters with significant differences in parameter specific sampling costs. In order to account for these challenges, we proposed the Bayesian optimisation method along with a dynamic scheduling function, which make a better balance of the parameter specific costs of sampling.

We performed feasibility tests of the proposed framework for the design problem of our hopping robot, and compare the performance against a human designer. In general the proposed design optimisation method work fairly well that it found optimal solutions in both 2-dimensional and 4dimensional design problems. The performance comparison against the human designer was also satisfactory in a sense that the proposed algorithm outperformed especially in the more challenging 4-dimensional optimisation problem.

Having said that, the results of the presented experimental results have to be interpreted with care. Due to the time constraints, we only conducted the experiments in a highly constrained manner. The optimisation performance depends on various factors, including the initialisation of the optimisation and choices of design parameters. In addition, human designers are likely to sample with different strategies, doubtlessly affecting the performance. A professional designer can make on average faster progress than a designer with limited understanding of mechanics and robotics. All of 

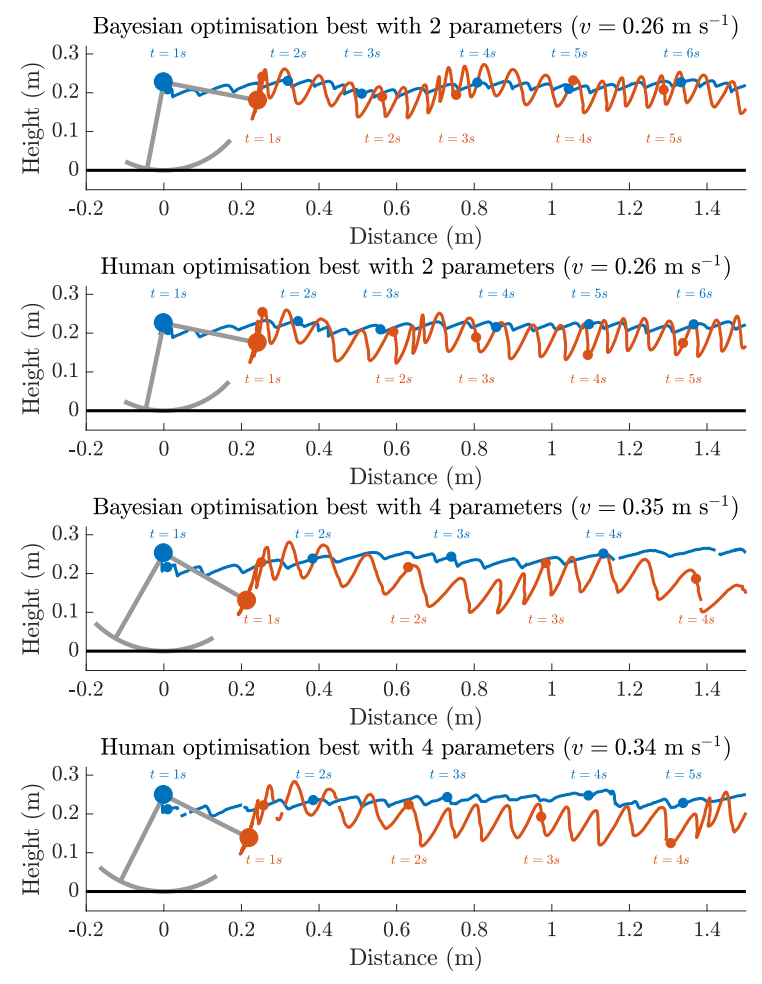

Fig. 7: Trajectories of CHIARO with different parameters obtained using Bayesian optimisation and human design process in 2-dimensional and 4-dimensional parameter space respectively. The red line shows the trajectory of the tip of the body and the blue line shows the trajectory of the joint. Small dots indicate time intervals of $1 \mathrm{~s}$, starting at $t=0 \mathrm{~s}$.

these unanswered questions need to be further investigated systematically in the future.

\section{ACKNOWLEDGEMENT}

The work described in this paper is supported in part by the U.K. Engineering and Physical Sciences Research Council under Grant EP/N03211X/1.

\section{REFERENCES}

[1] Chandana Paul and Josh C Bongard. The road less travelled: Morphology in the optimization of biped robot locomotion. In Intelligent Robots and Systems, 2001. Proceedings. 2001 IEEE/RSJ International Conference on, volume 1, pages 226-232. IEEE, 2001.

[2] Christine Chevallereau and Philippe Sardain. Design and actuation optimization of a 4-axes biped robot for walking and running. In Robotics and Automation, 2000. Proceedings. ICRA'O0. IEEE International Conference on, volume 4, pages 3365-3370. IEEE, 2000.

[3] Jeffrey Aguilar, Tingnan Zhang, Feifei Qian, Mark Kingsbury, Benjamin McInroe, Nicole Mazouchova, Chen Li, Ryan Maladen, Chaohui Gong, Matt Travers, Ross L Hatton, Howie Choset, Paul B Umbanhowar, and Daniel I Goldman. A review on locomotion robophysics: the study of movement at the intersection of robotics, soft matter and dynamical systems. Reports on Progress in Physics, 79(11):110001, 2016.

[4] Russ Tedrake, Teresa Weirui Zhang, and H Sebastian Seung. Stochastic policy gradient reinforcement learning on a simple 3d biped. In Intelligent Robots and Systems, 2004.(IROS 2004). Proceedings. 2004 IEEE/RSJ International Conference on, volume 3, pages 2849-2854. IEEE, 2004.
[5] C David Remy, Keith Buffinton, and Roland Siegwart. A matlab framework for efficient gait creation. In Intelligent Robots and Systems (IROS), 2011 IEEE/RSJ International Conference on, pages 190-196. IEEE, 2011

[6] John C Gallagher, Randall D Beer, Kenneth S Espenschied, and Roger D Quinn. Application of evolved locomotion controllers to a hexapod robot. Robotics and Autonomous Systems, 19(1):95-103, 1996.

[7] Karl Sims. Evolving virtual creatures. In Proceedings of the 21st annual conference on Computer graphics and interactive techniques, pages 15-22. ACM, 1994.

[8] Hod Lipson and Jordan B Pollack. Automatic design and manufacture of robotic lifeforms. Nature, 406(6799):974-978, 2000.

[9] Andre Rosendo, Marco von Atzigen, and Fumiya Iida. The trade-off between morphology and control in the co-optimized design of robots. PloS one, 12(10): e0186107, 2017.

[10] Vuk Vujovic, Andre Rosendo, Luzius Brodbeck, and Fumiya Iida Evolutionary developmental robotics: Improving morphology and control of physical robots. Artif Life, 23(2):169-185, Spring 2017.

[11] Luzius Brodbeck, Simon Hauser, and Fumiya Iida. Morphological evolution of physical robots through model-free phenotype development. PloS one, 10(6): $0128444,2015$.

[12] W. Roozing, Z. Li, D. G. Caldwell, and N. G. Tsagarakis. Design optimisation and control of compliant actuation arrangements in articulated robots for improved energy efficiency. IEEE Robotics and Automation Letters, 1(2):1110-1117, July 2016.

[13] Roberto Calandra, André Seyfarth, Jan Peters, and Marc Peter Deisenroth. Bayesian optimization for learning gaits under uncertainty. Annals of Mathematics and Artificial Intelligence, 76(1-2):5-23, 2016.

[14] Fabian Günther, Fabio Giardina, and Fumiya Iida. Self-stable onelegged hopping using a curved foot. In 2014 IEEE International Conference on Robotics and Automation (ICRA), pages 5133-5138. IEEE, 2014.

[15] Carl Edward Rasmussen. Gaussian processes for machine learning. Citeseer, 2006.

[16] Eric Brochu, Vlad M Cora, and Nando De Freitas. A tutorial on bayesian optimization of expensive cost functions, with application to active user modeling and hierarchical reinforcement learning. arXiv preprint arXiv:1012.2599, 2010.

[17] Dan Lizotte. Practical Bayesian Optimisation. PhD thesis, University of Alberta, 2008.

[18] Fabio Giardina and Fumiya Iida. Discrete foot shape changes improve dynamics of a hopping robot. In International Symposium on Experimental Robotics, pages 113-122. Springer, 2016.

[19] Jonathan Hunt, Fabio Giardina, Andre Rosendo, and Fumiya Iida Improving efficiency for an open-loop-controlled locomotion with a pulsed actuation. IEEE/ASME Transactions on Mechatronics, 21(3):1581-1591, 2016.

[20] Fabio Giardina, Fabian Guenther, and Fumiya Iida. Development and analysis of a self-stable, one-legged hopping robot. Master thesis of ETH Zurich, Switzerland, 2013.

[21] Jasper Snoek, Hugo Larochelle, and Ryan P Adams. Practical bayesian optimization of machine learning algorithms. In Advances in neural information processing systems, pages 2951-2959, 2012. 